\title{
Prognosis of Patients with Acute Myeloid Leukemia Regarding the Presence FLT3 Gene Mutation - a Case Report
}

\author{
Árpád Bzduch1, István Benedek Jr,2, Szilárd Bíró1, Johanna Sándor-Kéri1,2, Erzsébet Lázár ${ }^{1,2}$, \\ István Benedek ${ }^{1,2}$
}

${ }^{1}$ Clinic of Hematology and Bone Marrow Transplantation Unit, Tîrgu Mureş, Romania

2 University of Medicine and Pharmacy, Tîrgu Mureş, Romania

\section{CORRESPONDENCE}

István Benedek Jr

Str. Revoluției nr. 35

540042 Tîrgu Mureș, Romania

Tel: +40 265218739

E-mail: benedekistvan73@yahoo.com

\section{ARTICLE HISTORY}

Received: October 9, 2017

Accepted: November 16, 2017
Árpád Bzduch • Str. Revoluției nr. 35, 540042 Tîrgu Mureș, Romania. Tel: +40 265218739

Szilárd Bíró • Str. Revoluției nr. 35, 540042 Tîrgu Mureș, Romania. Tel: +40 265218739

Johanna Sándor-Kéri • Str. Revolutiei nr. 35, 540042 Tîrgu Mureș, Romania. Tel: +40 265218739

Erzsébet Lázár • Str. Revolutiei nr. 35, 540042 Tîrgu Mureș, Romania. Tel: +40 265218739

István Benedek • Str. Revoluției nr. 35, 540042 Tîrgu

Mures, Romania. Tel: +40 265218739

\begin{abstract}
Acute myeloid leukemia (AML) is a cancerous disease affecting the myeloid line of the bone marrow cells. FLT3, also known as CD135, is a proto-oncogene, which, if mutated, leads to different types of cancer. The protein it encodes presents tyrosine-kinase activity, and its intratandem mutation, FLT3-ITD, leads to uncontrolled proliferation of myeloblasts and worse outcomes in AML patients. There are currently several pharmacological agents that can inhibit the effect of either the proteins with tyrosine-kinase activity or the mutated FLT3 gene. We present the case of a 68-year-old patient, smoker, with a history of arterial hypertension, chronic obstructive pulmonary disease, presenting with headache unresponsive to antalgics, dyspnea after physical exertion, and epistaxis, with onset 2 months prior to his presentation. The patient was diagnosed with AML with positive FTL3 mutation for which conventional induction therapy was initiated. Within the next days, the patient presented several complications related to the disease itself or caused by the treatment, which eventually led to his death.
\end{abstract}

Keywords: acute myeloid leukemia, FTL3 mutation, stem cell transplantation

\section{INTRODUCTION}

Acute myeloid leukemia (AML) is a cancerous disease affecting the myeloid line of bone marrow cells. It occasionally presents as tumor masses in other tissues, such as the skin and lymph nodes, when it is called myeloid sarcoma. According to the FAB classification, there are several types of AML, more specifically: M0 - with minimal differentiation, M1 - without maturation, M2 - with maturation, M3 - acute promyelocytic leukemia, M4 - acute myelomonocytic leukemia, M5 - acute monoblastic leukemia, M6 - acute erythroleukemia, and M7 - acute megakaryoblastic leukemia. ${ }^{1,2}$

FLT3, also known as CD135, is a proto-oncogene, which, if mutated, leads to different types of cancer. This protein presents tyrosine-kinase activity, and its 
intra-tandem mutation, FLT3-ITD, leads to uncontrolled proliferation of myeloblasts. 3,4

The treatment of AML consists of chemotherapy, supportive treatment, and hematopoietic stem cell transplantation. Chemotherapy consists of two phases: remission induction and consolidation therapy. For remission induction, idarubicin $12 \mathrm{mg} / \mathrm{m}^{2}$ for 3 days and cytarabine 100 $\mathrm{mg} / \mathrm{m}^{2}$ is administered in combination, for a period of 7 days. In general, 2 cycles of this combination therapy are administered for a period of 3 to 4 weeks. The consolidation therapy consists in administrating $2-3$ cycles of highdose cytarabine $2000-3000 \mathrm{mg} / \mathrm{m}^{2}$ for 3 days. There are also remission induction therapies with other combinations, such as mitoxantrone $7 \mathrm{mg} / \mathrm{m}^{2}$ in days 1,3 , and 5 , with association of cytarabine $100 \mathrm{mg} / \mathrm{m}^{2}$ for 7 days. The FLAG-Ida combination therapy consists in the administration of fludarabine $30 \mathrm{mg} / \mathrm{m}^{2}$ for 5 days, with cytarabine $2000 \mathrm{mg} / \mathrm{m}^{2}$ for 5 days, idarubicin $10 \mathrm{mg} / \mathrm{m}^{2}$ for 3 days, and the administration of granulocyte-colony stimulating factor until the normalization of the neutrophil count. ${ }^{5-7}$

There are currently several pharmacological agents that can inhibit the effect of either the proteins with tyrosinekinase activity or the mutated FLT3 gene. ${ }^{8}$ Sorafenib is an oral medication that inhibits the effect of proteins with tyrosine-kinase activity, and it was initially developed for treating hepatocellular carcinoma or renal cell carcinoma. An additional effect of sorafenib is to inhibit the mutated FLT3 gene, thus having additional benefits in the treatment of AML. ${ }^{9}$ Quizartinib is a selective inhibitor of the FLT3-ITD mutation, and because of this, it represents an important substance in the treatment of AML with positive FLT3-ITD mutation. ${ }^{10}$ Midostaurin, an inhibitor of proteins that hold tyrosine-kinase activity, is also an inhibitor of FLT3-ITD, but its selectivity is much lower than that of quizartinib. ${ }^{11}$

\section{CASE PRESENTATION}

We present the case of a 68-year-old patient, smoker, with a history of arterial hypertension, chronic obstructive pulmonary disease, presenting with headache unresponsive to antalgics, dyspnea after physical exertion, and epistaxis, with onset 2 months prior to his presentation. The routine laboratory analysis showed high leucocyte count, anemia, and thrombocytopenia. The complete clinical examination revealed the presence of emphysematous thorax, crepitus rales at the lung bases, cardiomegaly, tachycardia (heart rate of 106/minute), and high blood pressure of 150/100 $\mathrm{mmHg}$.

The patient and the institution where the patient was admitted agreed to the publication of his data.
The patient underwent a bone marrow aspiration from the sternum for bone marrow analysis, which revealed the presence of $90 \%$ myeloblasts. The peripheral blood showed the presence of promyelocytes, anemia, thrombocytopenia, and absence of any kind of mature leucocyte. FLT3-ITD mutation and also MPL-RARa mutation were determined and were found to be positive. Patient management consisted in the initiation of remission induction therapy with idarubicin for 3 days and cytarabine for 7 days in adjusted doses, in association with all-trans retinoic acid $40 \mathrm{mg}$ per day, motivated by the presence of promyelocytes in the peripheral blood.

After 4 days of treatment, the patient presented lower leukocyte count, but also dyspnea, headache, and pain at the level of the sternum, associated with hemoptysis. These signs and symptoms arose the suspicion of disseminated intravascular coagulation (DIC), for which the D-dimer levels were evaluated and found at a level of $27.5 \mathrm{mg} / \mathrm{dL}$ (normal range $<0.5 \mathrm{mg} / \mathrm{L}$ ). Because of this, low-molecular-weight heparin was initiated, in association with transfusion and fresh frozen plasma as supportive therapy. Because of febrile neutropenia, antibiotic treatment was initiated with meropenem $3 \times 1 \mathrm{~g} /$ day, teicoplanin $2 \times 400 \mathrm{mg} /$ day, and colistin $4 \times 2 \mathrm{MIU} /$ day, as well as antiviral therapy with acyclovir $1,600 \mathrm{mg} /$ day and antifungal treatment with voriconazole $2 \times 200 \mathrm{mg} /$ day. After 7 days of treatment, the patient presented severe headaches resistant to analgesics, followed by motor impairment of the right side of his body, which raised the suspicion of a hemorrhagic stroke. The patient was transferred to the intensive care unit, where the hemorrhagic stroke was confirmed by cranial CT examination.

The supportive therapy was continued in the intensive care unit; however, after one week, the patient had deceased.

\section{DISCUSSIONS}

The presented case is part from a larger cohort of patients with AML that were admitted in the Hematology and Marrow Transplantation Clinic of Tîrgu Mureș between 2010 and 2017, 210 subjects more specifically, out of which 10 were found to be positive for FTL3-ITD mutation. From the total number of AML patients with positive FLT3-ITD mutation, none had survived, while from the total of 210 AML subjects, the survival rate is $7 \%(n=15)$.

A study conducted in Toronto, Canada, published in 2012, analyzed 97 new cases of AML over an 8-year period, out of which 70 presented relapse, and 57 were tested for FLT3-ITD mutation. All patients had received reinduc- 
tion therapy, 17 had undergone allogeneic bone marrow transplant, and 6 patients were positive for FLT3-ITD mutation. In total, 50 patients from the total of 70 relapse patients had deceased during the 9-month follow-up after relapse. ${ }^{12}$

In 2016, a case series on 4 patients with AML with FLT3 mutation was published, in which the patients were treated with chemotherapy, quizartinib, sorafenib, and allogeneic stem cell transplantation. One of the cases, a 48-year-old female patient, entered the remission phase after 4 cycles of chemotherapy, but presented relapse after 6 months for which she was treated with quizartinib and allogeneic stem cell infusion from a compatible donor. Despite having presented graft versus host disease involving the skin and liver for which she received prednisone and tacrolimus, and after a 90-day administration of sorafenib $200 \mathrm{mg}$ daily, the patient was still alive at the 5-year follow-up. The authors revealed that performing allogeneic stem cell transplantation as soon as possible after achieving remission leads to a significant improvement in overall survival and longer periods of remission. ${ }^{13}$ The patient reported in the present article was not treated with allogeneic stem cells, despite having a positive FLT3-ITD mutation, which could have been beneficial in this case.

A study performed by using data from the Center for International Blood and Marrow Transplant Research on 511 patients with de novo AML who had undergone hematopoietic stem cell transplantation found that $31 \%$ $(\mathrm{n}=158)$ of subjects were positive for FTL3 mutation, which led to a significantly higher risk of relapse compared to the wild-types ( $38 \%$ vs. $28 \%$, p $=0.04, \mathrm{RR}=1.6,95 \% \mathrm{CI}$ $1.15-2.22, \mathrm{p}=0.0048)$. However, the study found that the presence of FTL3 mutation was not associated with higher non-relapse mortality or overall survival, and $50 \%$ of subjects with this mutation who underwent stem cell therapy survived during the long-term follow-up. ${ }^{14}$

Another research on 481 patients with AML evaluated the impact of FTL3 mutations in three cytogenetic subgroups of patients: with core binding factor AML, with normal karyotype AML, and poor risk AML, respectively. The results showed no significant impact of the mutation in the first two groups, but in normal karyotype subjects FTL3-ITD mutations were associated with poorer outcomes, which were increasingly worse as the mutation burden was higher. ${ }^{15}$

In the above case report, the patient had been diagnosed with AML and was found to be positive for FTL3 mutation. Shortly after receiving conventional induction therapy with idarubicin and cytarabine, he presented disseminated intravascular coagulation, followed by a hemor- rhagic stroke, which eventually led to death. The relatively quick worsening of the patient's status did not give time for initiation of protocols for stem cell transplantation, which could have been a therapeutic alternative in this case.

\section{CONCLUSIONS}

Positive FLT3-ITD mutation leads to a poor prognosis of patients with acute myeloid leukemia, by leading either to increased mortality and complication rates, or to a high relapse rate. Allogeneic stem cell transplantation is a viable option for AML patients with or without FTL3 mutation, leading to better outcomes.

\section{CONFLICT OF INTEREST}

Nothing to declare.

\section{REFERENCES}

1. Arber DA, Orazi A, Hasserjian R, et al. The 2016 revision to the World Health Organization classification of myeloid neoplasms and acute leukemia. Blood. 2016;127:2391-2405.

2. Ghiaur G, Wroblewski M, Loges S. Acute Myelogenous Leukemia and its Microenvironment: A Molecular Conversation. Semin Hematol. 2015;52:200-206.

3. Kottaridis PD, Gale RE, Langabeer SE, et al. Studies of FLT3 mutations in paired presentation and relapse samples from patients with acute myeloid leukemia: implications for the role of FLT3 mutations in leukemogenesis, minimal residual disease detection, and possible therapy with FLT3 inhibitors. Blood. 2002;100:2393-2398.

4. Shih LY, Huang CF, Wu JH, et al. Internal tandem duplication of FLT3 in relapsed acute myeloid leukemia: a comparative analysis of bone marrow samples from 108 adult patients at diagnosis and relapse. Blood. 2002;100:2387-2392.

5. Cornelissen JJ, Blaise D. Hematopoietic stem cell transplantation for patients with AML in first complete remission. Blood. 2016;127:62-70.

6. Pefani E, Panoskaltsis N, Mantalaris A, Georgiadis MC, Pistikopoulos EN. Chemotherapy drug scheduling for the induction treatment of patients with acute myeloid leukemia. IEEE Trans Biomed Eng. 2014;61:2049-2056.

7. Kimby E, Nygren $P$, Glimelius B, et al. A systematic overview of chemotherapy effects in acute myeloid leukaemia. Acta Oncol. 2001;40:231-252

8. Gotink KJ, Verheul HMW. Anti-angiogenic tyrosine kinase inhibitors: what is their mechanism of action? Angiogenesis. 2010;13:1-14.

9. Man $\mathrm{CH}$, Fung TK, Ho C, et al. Sorafenib treatment of FLT3-ITD $(+)$ acute myeloid leukemia: favorable initial outcome and mechanisms of subsequent nonresponsiveness associated with the emergence of a D835 mutation. Blood. 2012;119:5133-5143.

10. Levis M. Quizartinib for the treatment of FLT3/ITD acute myeloid leukemia. Future Oncol. 2014;10:1571-1579.

11. Kayser S, Levis MJ, Schlenk RF. Midostaurin treatment in FLT3-mutated acute myeloid leukemia and systemic mastocytosis. Expert Rev Clin Pharmacol. 2017;10:1177-1189.

12. How J, Sykes J, Minden MD, et al. The prognostic impact of FLT3-ITD and NPM1 mutations in patients with relapsed acute myeloid leukemia and intermediate-risk cytogenetics. Blood Cancer J. 2013;3:e116.

13. Pratz KW, Levis M. How I treat FLT3-mutated AML. Blood. 2016;09:693648.

14. Deol A, Sengsayadeth S, Ahn KW, et al. Does FLT3 mutation impact survival after hematopoietic stem cell transplantation for acute myeloid leukemia? A Center for International Blood and Marrow Transplant Research (CIBMTR) analysis. Cancer. 2016;122:3005-3014.

15. Santos FP, Jones D, Qiao W, et al. Prognostic value of FLT3 mutations among different cytogenetic subgroups in acute myeloid leukemia. Cancer. 2011;117:2145-2155. 